
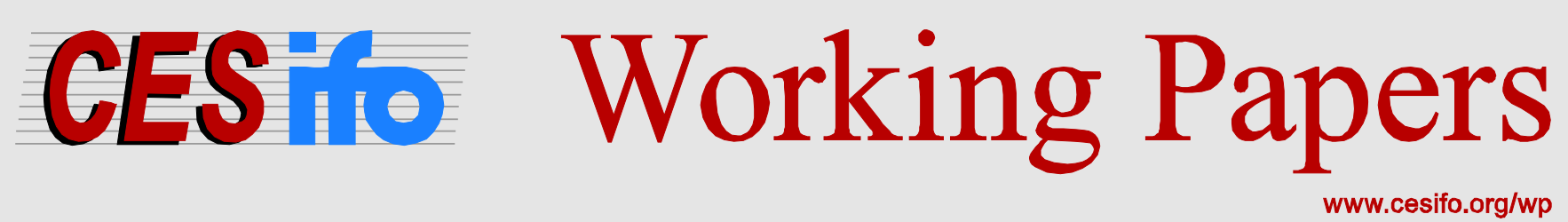

\title{
Cost Sharing in Collective Contests
}

\author{
Shmuel Nitzan \\ Kaoru Ueda
}

CESIFO WORKING PAPER NO. 4825

CATEgory 2: Public ChOICE

JUNE 2014

An electronic version of the paper may be downloaded

- from the SSRN website:

- from the RePEc website:

- from the CESifo website:

WWw.SSRN.com

www.RePEc.org

www.CESifo-group.org/wp

\section{CESifo}




\title{
Cost Sharing in Collective Contests
}

\begin{abstract}
This paper studies collective contests with endogenous cost sharing, general effort costs and intra-group heterogeneity of prize-valuation. Our objective is to clarify the relationship between cost sharing, intra-group heterogeneity within the competing groups and the elasticity of the marginal cost of effort incurred by the individual contestants. We also wish to stress the significance of intra-group heterogeneity in comparing the performance of cost sharing relative to prize sharing as a means of resolution of the collective action problem faced by the competing groups and present preliminary results for such comparisons. Our main results ascertain that unequal valuations of the contested prize within a group tend to result in a low degree of cost sharing. That is, intra-group heterogeneity prevents the group from establishing strong cost-sharing selective incentives.
\end{abstract}

JEL-Code: D700, D710, D720.

Keywords: collective contest, selective incentives, cost sharing, intra-group heterogeneity, elasticity of marginal effort costs, prize sharing.

\author{
Shmuel Nitzan* \\ Department of Economics \\ Bar Ilan University \\ Ramat Gan, 52900 / Israel \\ nitzans@mail.biu.ac.il
}

\author{
Kaoru Ueda \\ Faculty of Economics \\ Nanzan University \\ Nagoya, Aichi 466-8673 / Japan \\ k-ueda@ic.nanzan-u.ac.jp
}

*corresponding author

April 2014

Financial support from the Adar Foundation of the Economics Department at Bar-Ilan University is gratefully acknowledged by Shmuel Nitzan. 


\section{Introduction}

In a collective contest the contestants for a prize are groups. Applications of such contests include confrontations between labor unions and the employers, ethnic or religious conflicts, competition among academic institutes on quality-based recognition or on financial support, military conflict between countries, lobbying for trade protection, competitions on public facilities, and so on $^{4}$. Our main objective is to study the implications of intra-group heterogeneity on the choice of selective incentive devices. This paper focuses on one such device, cost sharing, which has been relatively less noticed. Recently Vázquez (2014) suggested the importance of cost sharing for a competing group as a means to enhance contributions by its members. As argued below, a transfer scheme within a group can be interpreted as cost sharing, provided that it depends on the sacrifices made by the individual group members to enhance their common interest. Such transfer schemes are often observed in the real world.

For example, more active members of a labor union confronting the employers are sometimes entitled to spend a higher amount of the fund collected as the union dues relative to less active members. In ethnic conflicts to establish some symbolic constructions or privileges, money and alternative resources are transferred to those directly and/or intensely engaged in the competition from their fellow associates. Competitions among universities or departments within the universities on reputation or on government financial support are usually determined on the basis of the demonstrated quality of the faculty members (publications, citations, etc.), which to some extent certainly depends on their exerted efforts. In such competing academic institutes, teaching obligations are sometimes inversely related to the quality and hence the efforts of the faculty members. In other words, in such cases, the transfer to the more active faculty members who are of a higher academic quality is made by the reduction in their teaching load. The above three practices can be conceived as actual transfer schemes (depending on the members' sacrifices) applied by groups competing on some kind of a prize.

When individuals win or lose the prize as a group, they have free-riding incentives in considering contribution to their group effort to enhance the probability of its winning. These incentives result in a typical collective-action problem, as

\footnotetext{
4 For more examples of contests in general, see Konrad (2009). On the basic theory of contests, see Hillman and Riley (1989) and Cornes and Hartley (2005).
} 
argued by Olson (1965). Collective contests could be viewed as a number of intragroup collective-action problems embedded in a competitive environment. Olson insists that in managing the problem a successful group relies on "selective incentives,” - incentives applied selectively to individuals depending on their actions. In the collective contests literature, a popular such device, which has been first studied in Nitzan $(1991)^{5}$, is commitment to a prize-sharing rule: prior to the contest, each competing group commits to a rule that divides part of the prize according to the members' efforts. The larger the part of the prize divided on the basis of the individuals' effort, the higher the selective incentive. ${ }^{6}$

When the contested prize is a group-specific public good, however, the device of prize sharing cannot be applied, at least in a straightforward way. ${ }^{7}$ It also does not work well when the prize is a group-specific commons openly accessible for all members of the winning group, which is the case studied in Nitzan and Ueda (2009). Even in such cases, however, a group could commit to a cost-sharing rule that imposes partial sharing of the cost of the members' sacrificed efforts. An individual who contributes more than others can then shift more cost to others, and as a result, get a net transfer. This is the reason why we can interpret the transfer schemes in the examples mentioned above as cost sharing. It works as another device of selective incentives even where the application of prize-sharing rules is problematic. And of course, it is also applicable in contests for private goods or impure public goods, where prize-sharing rules are applicable.

From the viewpoint of model building, cost-sharing rules have another advantage: intra-group heterogeneity can be easily incorporated. Individuals in a group are usually situated at various positions, politically, economically and culturally. Such intra-group heterogeneity can be reflected in their different valuations of the prize. In contrast, a group sharing rule, either prize sharing or cost sharing, operates impersonally and specifies a transfer to an individual depending only on his behavior. If individuals in a group are heterogeneous, their reactions to such a rule must be different. The question is whether such heterogeneity makes a group sharing rule more or less effective in overcoming the collective action problem. In this paper,

\footnotetext{
5 See Baik (1994), Lee (1995), and Ueda (2002) for early developments on this topic.

6 It should be noted that higher selective incentives are not necessarily better. When the members are rewarded for their effort, each member's effort has a negative externality for the others because their shares are cut. The result might be an excessive group effort. See Sen (1966).

7 This type of contests is firstly studied by Katz et.al. (1990). Also see Riaz et.al. (1995).
} 
we will shed new light on this problem by studying a model of a collective contest allowing both (endogenous) cost sharing and intra-group heterogeneity ${ }^{8}$. We find that unequal valuations of the prize within a group tend to result in a low degree of cost sharing. As will be argued, this result is explained by the observation that intra-group heterogeneity in valuations of the contested prize induces inefficient responses of the individuals to cost-sharing in a group.

In the next section, our model is introduced. Section 3 is devoted to the analysis of the equilibrium cost-sharing rules. Section 4 contains a preliminary examination of the relationship between prize-sharing rules and cost-sharing rules. Section 5 presents the conclusion.

\section{The Model}

\subsection{Group contests with cost sharing}

Consider $m$ groups competing for a prize. The number of individuals belonging to group $i$ is denoted by $N_{i}$. Each person individually and simultaneously decides how much contribute to enhance the win of his group. The individual contributions are aggregated in every group, and the probability of a group to win the prize is determined depending on those aggregated group efforts. Specifically, the win probability of group $i$ is assumed to be given by the lottery contest success function $\frac{A_{i}}{A}$, where $A_{i}$ is the effort of group $i$, and $A=\sum_{j=1}^{m} A_{j}$ is the total amount of effort by all competing groups. $a_{i k} \geq 0$ denotes the effort made by member $k$ of group $i$. We simply assume that $A_{i}=\sum_{k=1}^{N_{i}} a_{i k} \cdot{ }^{9}$ Each individual is assumed to be risk-neutral.

All members of a group have the same form of the effort cost function $c_{i}$, i.e. member $k$ of group $i$ has the cost $c_{i}\left(a_{i k}\right)$. It is a twice differentiable function with $c_{i}(0)=0, \quad c_{i}^{\prime}(a)>0 \quad$ and $\quad c_{i}^{\prime \prime}(a)>0 \quad$ for all $\quad a>0$. We also assume that $\lim _{a \rightarrow 0} c_{i}^{\prime}(a)=0{ }^{10}$. The valuation of the prize can be different among the group

\footnotetext{
${ }^{8}$ Vázquez (2014) has already tried to incorporate intra-group heterogeneity into his collective contest model with cost sharing. But his assumption of linear effort costs of individuals makes their behavior extreme (as in the earlier model of Baik (2008)); contributions are made only by those contestants with the maximal prize valuation. This peculiar equilibrium outcome hampers meaningful analysis of the relation between heterogeneity and cost sharing.

${ }^{9}$ See Kolmar and Rommeswinkel (2013) for contests with more general ways to aggregate efforts by individuals in a group.

${ }^{10}$ This assumption excludes the possibilities of non-contributors. As Nitzan and Ueda (2013) argue,
} 
members, reflecting their different positions within the group. The stake for the $k$ th individual belonging to the ith group is denoted by $v_{i k}>0$, which could be interpreted as the individual's valuation for the prize ${ }^{11}$. The distribution of the members' stakes in the contest can be represented by the stake vector of group $i, \mathbf{v}_{i}=\left(v_{i 1}, \ldots, v_{i N_{i}}\right)$.

Let us assume that part of the cost of the members' contributions is shared within the group. Specifically we assume that, in group $i, \delta_{i}\left(\delta_{i} \in[0,1]\right)$ of the effort cost of every member is compensated by making equal payback transfers that sum up to $\delta_{i}$ of the total exerted efforts. The cost of individual $k$ belonging to group $i$ has therefore the form

$$
\left(1-\delta_{i}\right) \cdot c_{i}\left(a_{i k}\right)+\delta_{i} \frac{\sum_{h=1}^{N_{i}} c_{i}\left(a_{i h}\right)}{N_{i}} \cdot 12
$$

And, in turn, the utility of member $k$ of group $i$ is given by

$$
E U_{i k}=\frac{A_{i}}{A} v_{i k}-\left\{\left(1-\delta_{i}\right) \cdot c_{i}\left(a_{i k}\right)+\delta_{i} \frac{\sum_{h=1}^{N_{i}} c_{i}\left(a_{i h}\right)}{N_{i}}\right\} \text {. }
$$

The degree of cost sharing, $\delta_{i}$, is determined in every group prior to the contest, to maximize the utilitarian group welfare (i.e. the sum of the expected utility (1) of all members of the group). This decision could be considered to be made (and implemented after the contest) by a benevolent group leader in each group. ${ }^{13}$ After observing the cost-sharing rule chosen by his own group, each member chooses the effort level individually.

Here, we assume that the decision on the degree of cost sharing in each group is unobservable from outside, as assumed by Baik and Lee (2007) and Nitzan and Ueda (2011), who consider the determination of the prize-sharing rules of competing groups in a contest. Both prize sharing and cost sharing result in redistributions made

such possibilities have important implications on the relation between the group performance and intragroup heterogeneity in terms of the stakes. The main concern of this paper is, however, the relation between the equilibrium cost-sharing rule and intra-group heterogeneity. Hence ignoring the possibility would be justified by the transparency of the analysis.

11 As discussed in Section 4, we can interpret it as the valuation of a mixed private-public-good prize. Then $v_{i k}$ is a function of two variables, the $k$ th individual's share of the private good prize and public good prize.

12 Notice that this function can be written as $c_{i}\left(a_{i k}\right)-\delta_{i} \frac{\sum_{h \neq k}\left(c_{i}\left(a_{i k}\right)-c_{i}\left(a_{i h}\right)\right)}{N_{i}}$, which implies that an individual who makes relatively larger contributions than others in the same group gets a net transfer. This is the way how cost sharing rules work as a device creating selective incentives.

13 Such an interpretation could be justified if the position itself is the intrinsic objective of the leaders, and the nomination requires the consensus of the group members. 
within the group, and the rules are applied only with respect to the insiders. They are changeable by notification only to the group members, and such changes could be made secretly, that is, without informing the persons belonging to the other groups. Even if a group sharing rule is openly announced to the outsiders, they would hardly believe that the announced rule is the final one the group really committed itself to. Hence it is doubtful whether the redistribution rules applied to the insiders can work as strategic devices for the opposing groups ${ }^{14}$. Even for cases where such observable commitment is possible, checking what happens if the sharing rules are unobservable is meaningful to reveal the pure strategic effects they have.

Hence we do not apply the usual two-stage-game formulation, in the first stage of which the cost-sharing rules are committed in each group and publicly known, and in the second stage of which the prize is contested. Instead, the contest under a configuration of cost-sharing rules in the competing groups is not a proper sub-game in our model. Every member in a group needs to infer the sharing rules of the other groups when he decides how much to contribute to the winning of the group. We therefore adopt the perfect Bayesian equilibrium notion as the solution concept of our model. Its precise description is given in the next subsection.

\subsection{Equilibrium}

We assume for simplicity that each player can use only pure strategies in equilibrium, and omit the possibility of randomization in the information sets. To characterize perfect Bayesian equilibrium, we need to describe the beliefs in the information sets of the players. Since the decisions on the cost-sharing rules by the leaders are made simultaneously at the beginning of the game, their beliefs are trivially given.

Hence let us consider the beliefs and the strategies of individuals in each of the competing groups, who make effort in the contest. Each information set of the $k$ th individual belonging to group $i$ can be indexed by a value of $\delta_{i}$ corresponding to the cost-sharing rule announced by the group leader. The individual cannot distinguish the nodes at which different sharing rules are chosen in other groups. A strategy of the member is, therefore, described as a function of only one variable $\delta_{i}$, which is denoted by $a_{i k}\left(\delta_{i}\right)$. Also, this individual's belief $\mu_{i k}$ with respect to the other groups' costsharing rules can depend on the value of $\delta_{i}$. Then $\mu_{i k}\left(\delta_{i}\right)$ is a probability measure

\footnotetext{
14 This problem is firstly pointed out by Katz (1991).
} 
defined on $[0,1]^{m-1}$, the space of possible configurations of the cost-sharing rules in the other groups $\delta_{-i}=\left(\delta_{1}, \cdots, \delta_{i-1}, \delta_{i+1}, \cdots, \delta_{m}\right)$.

Pick a profile of the sharing rules $\delta_{1}^{*}, \cdots, \delta_{m}^{*}$ and individual decisions on effort, $\left(a_{j h}^{*}\left(\delta_{j}\right), \mu_{j h}^{*}\left(\delta_{j}\right)\right)$, for all $\delta_{j} \in[0,1], j=1, \cdots, m$ and $h=1, \cdots, N_{j}$. Let us consider what conditions must hold if it is an equilibrium configuration. The expected utility of the individual $k$ belonging to group $i$ at the information set indexed by $\delta_{i}$ is calculated as

$$
v_{i k} \cdot \int\left(\frac{A_{i}^{*}\left(\delta_{i}\right)}{\sum_{j \neq i} A_{j}^{*}\left(\delta_{j}\right)+A_{i}^{*}\left(\delta_{i}\right)}\right) \mu_{i k}^{*}\left(\delta_{i}\right)\left(d \delta_{-i}\right)-\left\{\left(1-\delta_{i}\right) \cdot c_{i}\left(a_{i k}\left(\delta_{i}\right)\right)+\delta_{i} \cdot \frac{\sum_{h=1}^{N_{i}} c_{i}\left(a_{i h}\left(\delta_{i}\right)\right)}{N_{i}}\right\},
$$

where $A_{j}^{*}\left(\delta_{j}\right)=\sum_{h=1}^{N_{j}} a_{j h}^{*}\left(\delta_{j}\right)$. At the information set indexed by $\delta_{i}^{*}$, which lies on the equilibrium path, $\mu_{i k}^{*}\left(\delta_{i}^{*}\right)\left(\left\{\delta_{-i}=\delta_{-i}^{*}\right\}\right)=1$ holds by the requirement of consistency; the individuals must correctly infer the sharing rules in the other groups, given the strategies of the leaders. If we apply the "no-signaling-what-you-don't-know" condition of Fudenberg and Tirole (1991), we can also restrict the beliefs of the individuals in the information sets outside of the equilibrium path. This condition requires that choices of a group leader should not inform the group members anything about what she doesn't know. ${ }^{15}$ Since individuals in each group know that their own group leader cannot see any change of cost sharing in the other groups, they should consider that her deviations tell nothing new about this matter. At un-reached information set, therefore, the associated individual should keep the same belief as that held if the equilibrium group sharing rule is announced. The equilibrium belief by the $k$ th member of group $i, \mu_{i k}{ }^{*}$, must satisfy the condition

$$
\mu_{i k}^{*}\left(\delta_{i}\right)\left(\left\{\delta_{-i}=\delta_{-i}^{*}\right\}\right)=1 \text {, for all } \delta_{i} \neq \delta_{i}^{*} .
$$

Our solution concept for the model is such a pure-strategy perfect Bayesian equilibrium (with the “no-signaling-what-you-don't-know” condition).

The restrictions of the beliefs of the individuals discussed above make the characterization of their equilibrium strategy especially easy. The contribution by individual $k$ of group $i$ at the information set indexed by $\delta_{i}$ is the solution of

\footnotetext{
${ }^{15}$ The formal condition is given in Definition 6.1 of their paper.
} 


$$
\max _{a \geq 0} \frac{\sum_{h \neq k} a_{j h}^{*}\left(\delta_{i}\right)+a}{\sum_{j \neq i} A_{j}^{*}\left(\delta_{j}^{*}\right)+\sum_{h \neq k} a_{j h}^{*}\left(\delta_{i}\right)+a} v_{i k}-\left\{\left(1-\delta_{i}\right) \cdot c_{i}(a)+\delta_{i} \cdot \frac{\sum_{h \neq k} c_{i}\left(a_{j h}^{*}\left(\delta_{i}\right)\right)+c_{i}(a)}{N_{i}}\right\},
$$

and $a_{i k}^{*}\left(\delta_{i}\right)$ must satisfy the first-order condition

$$
\frac{\sum_{j \neq i} A_{j}^{*}\left(\delta_{j}^{*}\right)}{\left.\sum_{j \neq i} A_{j}^{*}\left(\delta_{j}^{*}\right)+A_{i}^{*}\left(\delta_{i}\right)\right\}^{2}} v_{i k}-\left\{\left(1-\delta_{i}\right)+\frac{\delta_{i}}{N_{i}}\right\} \cdot c_{i}^{\prime}\left(a_{i k}^{*}\left(\delta_{i}\right)\right)=0 \cdot \cdot^{16}
$$

This equation shows that the group leader can enhance the contribution of each member by raising the degree of cost sharing. It also implies the following useful relations holding between the contributions by the individuals belonging to the same group: ${ }^{17}$

$$
\frac{1}{v_{i k}} \cdot c_{i}^{\prime}\left(a_{i k}^{*}\left(\delta_{i}\right)\right)=\frac{1}{v_{i 1}} \cdot c_{i}^{\prime}\left(a_{i 1}^{*}\left(\delta_{i}\right)\right) \text { for all } \delta_{i} \in[0,1] \text { and } k=1, \cdots, N_{i} .
$$

Suppose that the leader of group $i$ induces the aggregate effort $A_{i}$ by controlling the degree of cost sharing. Since the marginal effort cost $c_{i}^{\prime}$ of group $i$ is assumed to be strictly increasing, the above relations uniquely determine the effort level of each individual belonging to the group. The sum of the effort cost of the group that induces the aggregate effort $A_{i}$ is, therefore,

$$
E_{i}\left(A_{i}\right)=\sum_{k=1}^{N_{i}} c_{i}\left(a_{i k}\left(A_{i}\right)\right)
$$

where $\sum_{k=1}^{N_{i}} a_{i k}\left(A_{i}\right)=A_{i}$ and $\frac{1}{v_{i k}} \cdot c_{i}^{\prime}\left(a_{i k}\left(A_{i}\right)\right)=\frac{1}{v_{i 1}} \cdot c_{i}^{\prime}\left(a_{i 1}\left(A_{i}\right)\right)$ for all $k=1, \cdots, N_{i}$.

This function tells the leader how much cost is generated in the group if she wants to induce a given level of aggregate group effort. As argued in the next section, it usually does not give the minimum sum of the group members' effort cost necessary to induce given aggregate effort $A_{i}$, because they voluntarily and individually choose their contributions. So we would refer to $E_{i}\left(A_{i}\right)$ as the distorted group cost function of group $i$.

Then the problem of the leader can be described as choosing the aggregate effort $A_{i}$ to maximize

$$
\frac{A_{i}}{\sum_{j=1}^{m} A_{j}} \sum_{k=1}^{N_{i}} v_{i k}-E_{i}\left(A_{i}\right)
$$

\footnotetext{
16 Because of the assumption $\lim _{a \rightarrow 0} c_{i}^{\prime}(a)=0$, the first-order condition for a maximum always holds as an equality.

17 A similar property is utilized by Ryvkin (2011).
} 
subject to the requirement that it can be realized by controlling the degree of cost sharing. This completes the description of equilibrium in our model.

To simplify the analysis, let us introduce the following condition.

Regularity Condition: The distorted group cost $E_{i}\left(A_{i}\right)$ is a convex function.

We do not step into technical details on what properties of the effort costs of individuals are needed to make this condition hold. But it is easy to confirm that two important cases that will be considered in the following sections satisfy it. The first case is that of intra-group homogeneity, i.e. all individuals in the same group have the same valuation of the prize; $v_{i k}=v_{i 1}$ for all $k=1, \cdots, N_{i}$. The second case is that of constant elasticity of marginal effort costs, in which $c_{i}(a)=\frac{K_{i}}{1+\alpha_{i}} a^{1+\alpha_{i}}$ for all $i=1, \cdots, m$, where $\alpha_{i}>0$ and $K_{i}>0$ are positive constants. A rather straightforward result of this regularity condition is the existence of equilibrium.

Proposition 1. If the above regularity condition holds, there exists a pure strategy perfect Bayesian equilibrium in our model of group contest with cost sharing.

Proof. See Appendix.

\section{Equilibrium Cost-Sharing Rules}

\subsection{Basic observations}

Now we can characterize the equilibrium rules of cost sharing chosen by the competing groups. It is convenient to introduce a new variable $\gamma_{i}=1-\delta_{i}+\frac{\delta_{i}}{N_{i}}$. Using the first order condition (2) to determine each group member's contribution, notice that it can be interpreted as the discount factor of marginal effort costs reflecting cost sharing. Since this factor is strictly decreasing in $\delta_{i}$, we can argue on the equilibrium cost sharing scheme of group $i$ via the value of $\gamma_{i}$ instead of $\delta_{i}$. It takes the value $\frac{1}{N_{i}}$ under the full sharing costs $\left(\delta_{i}=1\right)$, and the value 1 under no sharing costs ( $\left.\delta_{i}=0\right)$. By using the properties of $E_{i}\left(A_{i}\right)$ and the first-order condition to maximize 
the function (4), we can derive the basic result on the equilibrium cost-sharing rules;

Proposition 2. In equilibrium, the cost-sharing scheme chosen by group $i$ is characterized by the equation

$$
\gamma_{i}=\sum_{k=1}^{N_{i}} \frac{v_{i k}}{\sum_{p=1}^{N_{i}} v_{i p}} \cdot \frac{v_{i k} / c_{i}^{\prime \prime}\left(a_{i k}^{*}\right) .}{\sum_{p=1}^{N_{i}}\left(v_{i p} / c_{i}^{\prime \prime}\left(a_{i p}^{*}\right)\right)},
$$

as long as it belongs to $\left[1 / N_{i}, 1\right]$, where $a_{i k}^{*}$ is the equilibrium contribution by individual $k$ belonging to group $i$.

Proof. See Appendix.

It should be clear that the right-hand-side of equation (5) is strictly less than one. The next corollary directly follows:

Corollary 1. Under the pure strategy Bayesian perfect equilibrium of the model, at least some degree of cost sharing is implemented in every group.

Hence a competing group always adopts cost sharing if it is possible, to give selective incentives to its members.

Also, we can directly derive the cost-sharing rule in the case of intra-group homogeneity, i.e. every individual in the group has the same valuation of the prize, which is the starting point to analyze the effect of intra-group heterogeneity on cost sharing. Condition (3) requires that contestants with the same stake will choose the same level of effort. This fact combined with Proposition 2 gives the next result.

Corollary 2. When all group members' stakes are equal, the cost is fully shared.

It is not difficult to understand how this clear-cut result is derived ${ }^{18}$. Assume that a group is homogeneous in the sense that all members have the same valuation of

\footnotetext{
18 In Vázquez (2014), cost sharing rules that are observable from outside work as strategic variables. This is the reason why in his model the disadvantaged group does not choose full cost sharing even though it has homogeneous members.
} 
the prize, i.e. $v_{i k}=v$ for all $k=1, \cdots, N_{i}$. By condition (3), they will choose the same effort level. Then $E_{i}\left(A_{i}\right)=N_{i} \cdot c_{i}\left(\frac{A_{i}}{N_{i}}\right)$, and the condition characterizing the aggregate group effort maximizing the group welfare (4) is

$$
\frac{\sum_{j \neq i} A_{j}}{\left(\sum_{j=1}^{m} A_{j}\right)^{2}} \cdot v=\frac{1}{N_{i}} \cdot c_{i}^{\prime}\left(\frac{A_{i}}{N_{i}}\right) \text {. }
$$

If such level of aggregate group effort is realizable with a possible cost-sharing rule, this is what the group leader will choose. Since condition (2) relating the cost-sharing rule to each member's contribution has the form

$$
\frac{\sum_{j \neq i} A_{j}}{\left(\sum_{j=1}^{m} A_{j}\right)^{2}} \cdot v-\left\{\left(1-\delta_{i}\right)+\frac{\delta_{i}}{N_{i}}\right\} \cdot c_{i}^{\prime}\left(\frac{A_{i}}{N_{i}}\right)=0,
$$

in this intra-group homogeneity case, the desired effort level satisfying equation (6) is attained by setting $\delta_{i}=1$.

\subsection{Cost sharing and intra-group heterogeneity.}

We have a benchmark result that the full sharing of the cost is best for a group when all of its members have the same stake from the contested prize. But it does not directly imply that increased similarity in the gains from the prize in a group enhances the degree of cost sharing. What is then the relationship between the distribution of the stakes in a group and the degree of cost sharing? If the class of effort cost functions is confined to the form of constant elasticity of marginal costs, i.e., $c_{i}(a)=K_{i} \cdot \frac{a^{1+\alpha_{i}}}{1+\alpha_{i}}\left(\alpha_{i}>0\right)$, we find a surprisingly tractable answer to this interesting question, which is also very suggestive for considering more general cases.

Proposition 3. Assume that the effort cost function of every member of group i has the form of constant elasticity of marginal costs, $c_{i}(a)=K_{i} \cdot \frac{a^{1+\alpha_{i}}}{1+\alpha_{i}}\left(\alpha_{i}>0, K_{i}>0\right)$. Then, the equilibrium cost- sharing rule satisfies the following equation;

$$
\gamma_{i}=\frac{1}{\sum_{p=1}^{N_{i}} v_{i p}} \sum_{k=1}^{N_{i}} \frac{v_{i k}^{\frac{1}{\alpha_{i}}+1}}{\sum_{p=1}^{N_{i}} v_{i p}^{\frac{1}{\alpha_{i}}}} .
$$


Proof. See Appendix.

For an $n$-dimensional vector $\mathbf{v}=\left(v_{1}, \ldots, v_{n}\right)$, the Lehmer mean with index $q$ is defined by $\quad L_{n}(\mathbf{v}, q)=\sum_{k=1}^{n} \frac{v_{k}^{q}}{\sum_{p=1}^{n} v_{p}^{q-1}} \quad$ (Lehmer (1971)). Proposition 3 implies that the equilibrium cost-sharing scheme can be represented using this generalized mean as follows;

$$
\gamma_{i}=\frac{L_{N_{i}}\left(\mathbf{v}_{i}, \frac{1}{\alpha_{i}}+1\right)}{\sum_{p=1}^{N_{i}} v_{i p}} .
$$

The following known properties of the Lehmer mean are useful in analyzing the equilibrium cost-sharing rules:

(i) For a given $\mathbf{v}, L_{n}(\mathbf{v}, q)$ is strictly increasing in $q$,

(ii) $\quad L_{n}(\mathbf{v}, 1)=\frac{\sum_{k=1}^{n} v_{k}}{n}$, and

(iii) $\lim _{q \rightarrow \infty} L_{n}(\mathbf{v}, q)=\max \left\{v_{1}, \ldots, v_{n}\right\}$.

By the above properties we directly get the following results on the relation between the cost-sharing schemes and the elasticity of marginal costs.

Corollary 3. Assume that the effort cost function of an individual in group $i$ has the form $c_{i}(a)=K_{i} \cdot \frac{a^{1+\alpha_{i}}}{1+\alpha_{i}} \quad\left(\alpha_{i}>0\right)$. Then, given $\mathbf{v}_{i}$, the degree of cost-sharing in equilibrium is strictly increasing in $\alpha_{i}$. Furthermore, we have the two limit cases on the equilibrium discount factor of marginal effort costs, $\lim _{\alpha_{i} \rightarrow \infty} \gamma_{i}=\frac{1}{N_{i}}$ and $\lim _{\alpha_{i} \rightarrow 0} \gamma_{i}=\frac{\max \left\{v_{i 1}, \ldots, v_{i N_{i}}\right\}}{\sum_{k=1}^{N_{i}} v_{i k}}$.

Hence, given a distribution of stakes in a group, the higher the elasticity of marginal costs, the higher the implemented degree of cost sharing. Since a larger elasticity of marginal costs discourages every group member's contribution, the group would rely more on cost sharing to counteract the negative effect. The value of $\lim _{\alpha_{i} \rightarrow \infty} \gamma_{i}$ shows 
that such stimulus finally results in the full sharing of effort cost.

One may feel that the other limit, $\lim _{\alpha_{i} \rightarrow 0} \gamma_{i}$, has a strange form ${ }^{19}$. Actually this value coincides with that derived from the optimal cost-sharing rule in the case of constant marginal costs, that is, $\alpha_{i}=0$ or $c_{i}(a)=K_{i} \cdot a$, which is the limit (but unincluded) case of our model. With constant marginal costs, only the contestants with the largest stake in the group are active, as established in Baik (2008). In this case the aggregate effort of group $i, A_{i}$, is determined by the equation

$$
\frac{A-A_{i}}{A^{2}} \cdot \max \left\{v_{i 1}, \ldots, v_{i N_{i}}\right\}=\gamma_{i} \cdot K_{i} \cdot
$$

It can be shown that the group effort maximizing the utilitarian group welfare is then determined by the equation

$$
\frac{A-A_{i}}{A^{2}} \cdot \sum_{k=1}^{N_{i}} v_{i k}=K_{i},
$$

which directly leads to the above value of $\lim _{\alpha_{i} \rightarrow 0} \gamma_{i}$.

Let us turn to the connection between the distribution of the stakes and the degree of cost sharing. By equation (7), the discount factor of marginal effort costs $\gamma_{i}$ coincides with a familiar index when the cost function is quadratic, i.e. $\alpha_{i}=1$;

$$
\gamma_{i}=\frac{L_{N_{i}}\left(\mathbf{v}_{i}, 2\right)}{\sum_{p=1}^{N_{i}} v_{i p}}=\sum_{k=1}^{N_{i}}\left(\frac{v_{i k}}{\sum_{p=1}^{N_{i}} v_{i p}}\right)^{2} .
$$

The right-hand-side is the Herfindahl-Hirschman Index (HHI). In this case the degree of cost sharing $\delta_{i}$ directly declines with the inequality of stakes in a group measured by the HHI. This observation suggests that, at least in the cases of constant elasticity of marginal costs, cost sharing should be loosened up as the distribution of stakes gets more unequal.

To examine this conjecture, we will use the concept of Lorenz-dominance to determine whether a stake vector is more unequal (or "less nearly equal") than another vector. Take two stake vectors $\mathbf{v}=\left(v_{1}, \ldots ., v_{n}\right)$ and $\mathbf{v}^{\prime}=\left(v_{1}^{\prime}, \ldots ., v_{n}^{\prime}\right)$, and assume that, without loss of generality, $v_{1} \leq \ldots \leq v_{n}$ and $v_{1}^{\prime} \leq \ldots \leq v_{n}^{\prime}$. The latter vector (distribution of group members' prize valuations) is called more unequal than the

\footnotetext{
19 Since $\max \left\{v_{i 1}, \ldots, v_{i N_{i}}\right\}$ is larger than or equal to the arithmetic mean of the stakes of the individuals, $\lim _{\alpha_{i} \rightarrow 0} \gamma_{i}$ is in fact larger than or equal to $1 / N_{i}$.
} 
former in the sense of Lorenz-dominance, if $\sum_{k=1}^{h} v_{k} \geq \sum_{k=1}^{h} v_{k}^{\prime}$ for all $h \leq n$, with strict inequality for at least one $h$, and $\sum_{k=1}^{n} v_{k}=\sum_{k=1}^{n} v_{k}^{\prime 20}$. It is equivalent to say that $\mathbf{v}$ can be obtained from $\mathbf{v}^{\prime}$ by a finite sequence of transformations (called the Dalton transfers) of the form $v_{i l}(t+1)=v_{i l}(t)+\varepsilon(t) \leq v_{\text {ih }}(t), \quad v_{\text {ih }}(t+1)=v_{\text {ih }}(t)-\varepsilon(t) \geq v_{\text {il }}(t)$ with $\varepsilon(t)>0$ for some $h$ and $l(h>l)$, and $v_{i k}(t+1)=v_{i k}(t)$ for all $k \neq h, l$, where at least in one of the transformations the inequality must be strict. It is an easy calculation to confirm the following results regarding the relationship between the Lehmer mean and this concept of inequality.

Lemma 1. Let $\mathbf{v}_{i}^{\prime}=\left(v_{i 1}^{\prime}, \ldots ., v_{i n}^{\prime}\right)$ be a vector more unequal than $\mathbf{v}_{i}=\left(v_{i 1}, \ldots ., v_{i N_{i}}\right)$ in the sense of Lorenz-dominance. Then, for $2 \geq q>0, L_{n}\left(\mathbf{v}_{i}^{\prime}, q\right)>L_{n}\left(\mathbf{v}_{i}, q\right)$.

With this proposition, we can argue as follows; suppose that a competing group $i$ has the stake vector $\mathbf{v}_{i}=\left(v_{i 1}, \ldots ., v_{i N_{i}}\right)$, and the effort cost functions of the members have the form $c_{i}(a)=K_{i} \cdot \frac{a^{1+\alpha_{i}}}{1+\alpha_{i}}$. By Proposition 3, the equilibrium costsharing rule is characterized by the equation $1-\delta+\frac{\delta}{N_{i}}=\frac{L_{N_{i}}\left(\mathbf{v}_{i}, \frac{1}{\alpha_{i}}+1\right)}{\sum_{p=1}^{N_{i}} v_{i p}}$, where $\delta$ is the degree of cost sharing. If $\alpha_{i} \geq 1$, or the marginal cost function is convex, then the more unequal stake vector in the sense of Lorenz dominance the group has, the lower the degree of cost sharing it applies.

When the marginal cost function is strictly concave or $0<\alpha_{i}<1$, we do not obtain a clear-cut result as in the case where $\alpha_{i} \geq 1$. Since $L_{N_{i}}\left(\mathbf{v}_{i}, \frac{1}{\alpha_{i}}+1\right)$ is decreasing with $\alpha_{i}$, however, the value $1-\delta+\frac{\delta}{n}$ is never lower than the HHI in this case. The HHI goes up and approaches 1 as the stake vector becomes more unequal ${ }^{21}$. Thus the value of $1-\delta+\frac{\delta}{n}$ asymptotically rises as the stake vector gets worse in the sense of

\footnotetext{
${ }^{20}$ If the condition "with strict inequality for at least one $h$ " is dropped, we have the definition of majorization, and then it is said that $\mathbf{v}_{i}^{\prime}=\left(v_{i 1}^{\prime}, \ldots, v_{i N_{i}}^{\prime}\right)$ majorizes $\mathbf{v}_{i}=\left(v_{i 1}, \ldots ., v_{i N_{i}}\right)$.

${ }^{21}$ Since Lorenz-dominance is a partial ordering relation, we would need to adequately specify a sequence of stake vectors.
} 
Lorenz dominance, even when $0<\alpha_{i}<1$. As a whole, we could say that the conjecture made at the beginning of this subsection is correct;

Proposition 4. Assume that the effort cost function of an individual in group i has the form $c_{i}(a)=K_{i} \cdot \frac{a^{1+\alpha_{i}}}{1+\alpha_{i}}\left(\alpha_{i}>0\right)$. When $\alpha_{i} \geq 1$, a more unequal stake vector in the sense of Lorenz dominance results in a lower degree of cost sharing. Even when $0<\alpha_{i}<1$, the degree of cost sharing asymptotically declines as the distribution of stakes becomes more unequal.

\subsection{Distorted group costs and intra-group heterogeneity.}

Why does heterogeneity of stakes in a group reduce the equilibrium degree of cost sharing? Distributional concerns for the net benefit of group members would play a minor role in choices of a group leader, because her objective is assumed to be the utilitarian group welfare. We should seek the answer in the realm of the efficiency problem faced by the group.

Intra-group heterogeneity of valuations of the prize truly causes inefficient allocation of contributions among the individual group members. In the context of a multi-plant firm, we are aware of an elementary result: it should equate the marginal costs of its plants to minimize the cost of producing a given amount of output. The same is true for a multi-member contesting group: it should equate the marginal effort costs of its members to minimize the total cost of putting a given amount of the aggregate group effort. If the members are homogeneous, the individuals spontaneously realize such bearing of contributions through condition (3). Otherwise, their voluntary contributions do not equate the marginal effort costs. Hence the distorted group cost function, $E_{i}\left(A_{i}\right)$, mentioned in Section 2, does not minimize the total cost needed to induce a given level of aggregate group effort unless the group members have the same valuation of the prize.

Let us consider this point precisely. Between individuals $h$ and $l$ with $v_{i l}<v_{i h}$, the gap of the marginal effort costs

$$
c_{i}^{\prime}\left(a_{i h}^{*}\left(\delta_{i}\right)\right)-c_{i}^{\prime}\left(a_{i l}^{*}\left(\delta_{i}\right)\right)=\left(\frac{v_{i h}}{v_{i l}}-1\right) \cdot c_{i}^{\prime}\left(a_{i h}^{*}\left(\delta_{i}\right)\right)
$$

occurs at the degree of cost sharing $\delta_{i}$, by equation (3). Because of such differences 
of marginal effort costs among the members, heterogeneous groups experience inefficiency in exerting a given level of aggregate effort. Enhancing the cost sharing, the members increase the contribution keeping the above equation. The discrepancies between the members' marginal effort costs are therefore increased, and the inefficiency in contributions gets worse. This effect piles up the extra burden to enhance group effort, causing the leader to hesitate when considering the application of a high degree of cost sharing.

Actually, in the case of constant elasticity of marginal costs, we can directly calculate the marginal distorted group cost $E_{i}^{\prime}\left(A_{i}\right)$ to get that

$$
E_{i}^{\prime}\left(A_{i}\right)=\frac{K_{i}}{\left(\sum_{p=1}^{N_{i}} \frac{1}{v_{i p}}\right)^{\alpha_{i}}} \cdot L_{N_{i}}\left(\mathbf{v}_{i}, \frac{1}{\alpha_{i}}+1\right) \cdot A^{\alpha_{i}}
$$

When $\alpha_{i} \geq 1,\left(\sum_{p=1}^{N_{i}} v_{i p}^{\frac{1}{\alpha_{i}}}\right)^{\alpha_{i}}$ is a concave CES function with respect to the stake vector. Hence it increases with a Lorenz-dominating change of the stake vector ${ }^{22}$. We can see that the marginal distorted group cost gets higher as the stake vector becomes more unequal, reflecting increased inefficiency as have been pointed out above.

\subsection{The effect of intra-group heterogeneity on cost conditions}

To confirm that the logic we have explained in the last subsection is an essential factor in preventing full sharing of costs in a group, let us resort to the following argument. Keeping all other components of our model, individual valuations of the prize and the cost functions are changed as follows; the cost function of individual $k$ belonging to group $i$ becomes $\frac{1}{v_{i k}} \cdot c_{i}\left(a_{i k}\right)$, and the valuation of the prize is just 1 . Then, his equilibrium choice of contribution satisfies the modified first-order condition

$$
\frac{\sum_{j \neq i} A_{j}^{*}\left(\delta_{j}^{*}\right)}{\left\{\sum_{j \neq i} A_{j}^{*}\left(\delta_{j}^{*}\right)+A_{i}^{*}\left(\delta_{i}\right)\right\}^{2}}-\left\{\left(1-\delta_{i}\right)+\frac{\delta_{i}}{N_{i}}\right\} \cdot \frac{1}{v_{i k}} \cdot c_{i}^{\prime}\left(a_{i k}^{*}\left(\delta_{i}\right)\right)=0,
$$

which replaces equation (2) in the original model. It is obvious that this condition and

22 Dasgupta et.al. (1973). 
equation (2) result in the same reaction to any given degree of cost sharing, under any combination of the values $v_{i k}$ 's. If intra-group heterogeneity can be represented as a distribution of those $v_{i k}$ 's, the relation between intra-group heterogeneity and the induced contributions by cost sharing is the same as in the original setting. However, individual contributions no longer cause inefficiency here, even though the $v_{i k}$ 's are different within the group; heterogeneous individuals in a group contribute differently because of their different technologies, i.e., cost functions, but their marginal costs in equilibrium are now always equal. This modified model can be referred to as the associated contest with intra-group heterogeneous cost functions. In such an associated contest, how do the group leaders choose the degree of cost sharing? Actually, we have the following result:

Proposition 5. In equilibrium of the associated contest with intra-group heterogeneous cost functions, the individuals in a group fully share their costs.

Proof. See Appendix.

That is, in the modified setting the cost is fully shared. How much contributions can be induced from heterogeneous individuals is, therefore, not critical for a group leader in avoiding full cost sharing. The created inefficiency makes the difference.

\section{Prize Sharing versus Cost Sharing}

Prize sharing is another type of incentive schemes intended to mitigate collective action problems. Undoubtedly, it has been studied more intensely than cost sharing in the literature of collective contests. It seems natural to ask how the outcomes of the two types of sharing rules differ. Since prize sharing works through the private-good part of the prize, it would be better to explicitly specify the privategood and the public-good parts of the prize. Let every member of every group have the same benefit function $B(q, G)$ specifying the value of the prize, where $q$ is the amount of the private good and $G$ is the amount of the group-specific public good. This function is twice differentiable, and $B(q, G)>0$, unless $(q, G)=(0,0)$. Furthermore, $\frac{\partial B}{\partial q}(q, G)>0, \frac{\partial B}{\partial G}(q, G) \geq 0$, and $\frac{\partial^{2} B}{\partial q^{2}}(q, G) \leq 0$ hold for all $q>0, G>0$. 
A prize-sharing rule is assumed to be a linear combination of the egalitarian and the relative effort rules applied to the private-good part of the prize. The weight of the relative effort rule, $\theta_{i}$, is determined in every group prior to the contest, to maximize the utilitarian group welfare. The rule is unobservable from outside, as we have assumed regarding the cost-sharing rules. Denote the total amount of the privategood prize by $M>0$. When the group wins, the member having put effort $a \geq 0$ receives the following amount of the private good;

$$
\left(\theta_{i} \cdot \frac{a}{A_{i}}+\left(1-\theta_{i}\right) \cdot \frac{1}{N_{i}}\right) \cdot M .
$$

We could call this model of collective contests with unobservable prize-sharing rules, which is examined by Nitzan and Ueda (2011), the prize-sharing contests for a mixture of public and private goods. On the other hand, it is plausible to assume that in contests with (unobservable) cost-sharing schemes, the private-good part of the prize is equally divided in the winning group. Let us call such contests the costsharing contests for a mixture of public and private goods.

The cost-sharing contests can be treated as a special case of our main model because we can set $v_{i k}=B\left(\frac{M}{N_{i}}, P_{i k}\right)$ for individual $k$ of group $i . P_{i k}$ is the valuation of the public-good prize, which possibly differs among individuals. In either type of contests for a mixture of public and private goods, intra-group heterogeneity occurs by different valuations of the public-good part of the prize. For prize-sharing contests, we can apply the arguments made by Nitzan and Ueda (2011, Lemma 1) to derive the observation that, in a pure strategy perfect Bayesian equilibrium, individual $k$ of the group $i$ chooses the contribution $a_{i k}$ satisfying

$$
\frac{\sum_{j \neq i} A_{j}}{\left(\sum_{j=1}^{m} A_{j}\right)^{2}} B\left(\left(\theta_{i} \cdot \frac{a_{i k}}{A_{i}}+\left(1-\theta_{i}\right) \cdot \frac{1}{N_{i}}\right) \cdot M, P_{i k}\right)+\frac{1}{\sum_{j=1}^{m} A_{j}} \cdot \frac{A_{i}-a_{i k}}{A_{i}} \cdot \theta_{i} M \cdot \frac{\partial B}{\partial q}-c_{i}^{\prime}\left(a_{i k}\right)=0 .
$$

To begin with, consider the case of intra-group homogeneity, i.e. every individual in the same group has the same valuation of the public-good part of the prize; $P_{i k}=P_{i}$ for all $k=1, \cdots, N_{i}$ and all $i=1, \cdots, m$ (the prize can be a pure private goods where $P_{i}=0$ ). We have shown, in sub-section 3(a), that every individual in the same group chooses the same effort level, and each group adopts full cost sharing ( $\left.\delta_{i}=1, i=1, \cdots, m\right)$ in such a case of cost-sharing contests. The aggregate effort $A_{i}$ and 
each individual's contribution $\frac{A_{i}}{N_{i}}$ are therefore determined by the equation $\frac{\sum_{j \neq i} A_{j}}{\left(\sum_{j=1}^{m} A_{j}\right)^{2}} \cdot B\left(\frac{M}{N_{i}}, P_{i}\right)=\frac{1}{N_{i}} \cdot c_{i}^{\prime}\left(\frac{A_{i}}{N_{i}}\right)$, which corresponds to equation (6).

Now, suppose that a prize-sharing rule for group $i$ characterized by some value of $\theta_{i}$ can induce the same configuration of the members' contributions as under the full-cost sharing. What conditions does it satisfy? By using (10), we can show that every individual belonging to the same group chooses the same effort level for a prize-sharing rule, if intra-group homogeneity holds. In such a case, an individual of group $i$ chooses the effort level $\frac{A_{i}}{N_{i}}$ satisfying the equation

$$
\frac{\sum_{j \neq i} A_{j}}{\left(\sum_{j=1}^{m} A_{j}\right)^{2}} B\left(\frac{M}{N_{i}}, P_{i}\right)+\frac{1}{\sum_{j=1}^{m} A_{j}} \cdot\left(1-\frac{1}{N_{i}}\right) \cdot \theta_{i} M \cdot \frac{\partial B}{\partial q}-c_{i}^{\prime}\left(\frac{A_{i}}{N_{i}}\right)=0 .
$$

By the above two equations and some algebraic manipulations, we can derive the following condition for $\theta_{i}$;

$$
\theta_{i}=\left(1-\frac{A_{i}}{A}\right) \cdot \frac{1}{\eta\left(M / N_{i}, P_{i}\right)},
$$

where $\eta\left(M / N_{i}, P_{i}\right)=\frac{\partial}{\partial q} B\left(M / N_{i}, P_{i}\right) \cdot \frac{M / N_{i}}{B\left(M / N_{i}, P_{i}\right)}$ is the elasticity of the benefit from the private-good part of the prize. The prize-sharing rule characterized by this $\theta_{i}$ coincides with the equilibrium prize-sharing rule of a prize-sharing contest under intra-group homogeneity, that has been established in Nitzan and Ueda (2011, Proposition 1). ${ }^{23}$

Thus we can see that, under intra-group homogeneity, the equilibrium effort by every individual in a group is the same in both the cost-sharing contest and the

\footnotetext{
${ }^{23}$ Equation (11) tells that, if two competing groups in a prize-sharing contest are of the same size and the individuals have the same valuation of the public good prize in both of the groups, the group attaining the lower winning probability is the one that divides a larger part of the prize according to the relative effort rule (See Nitzan and Ueda (2011) for the details). It is interesting that this seemingly strange pattern is equivalent to the full cost-sharing by all groups. Once we notice that the pattern is caused by the incentives depending not on the costs but on the prize, it becomes more understandable; strong incentives are needed if the win probability is low because the reward for the contribution is less probable. Strong incentives are also needed if the elasticity of the benefit from the private-good part of the prize is low, because the reward is less tempting.
} 
prize-sharing contest. The next proposition is straightforward:

Proposition 6. Let every individual belonging to the same group have the same valuation of the public-good part of the prize in a contest for a mixture of public and private goods. Then, the prize-sharing contests and the cost-sharing contests result in the same equilibrium configuration of contributions by individuals.

Hence, as long as the individuals in the same group have the same valuation of the public-good prize, the results on the individuals' effort levels in one of the sharing modes are also true for the other. For example, we can use a result of Nitzan and Ueda (2011, Corollary 1) to confirm that, if every individual in every group has the same valuation of the public good prize $P>0$, a larger group always attains a higher win probability in the cost-sharing contest.

We can understand the reason for this equivalence by using the observations made in sub-section 3(c) of our main model. Recall that the efficient bearing of aggregate effort by the members of a group is realized if the valuation of the prize is the same for all of them. The distorted group cost $E_{i}\left(A_{i}\right)$ coincides with the true group cost function that gives the minimal total effort cost for the group necessary for inducing the aggregate effort $A_{i}$. Therefore, when the function (4) is maximized by full-cost sharing, the resulting configuration of contributions by individuals coincides with the first best contributions, in the sense that the leader would assign it to the individuals in the group if she could directly determine their contributions. This is also true for the cost-sharing contest discussed in this section, which is a special case of the main model. If the leader can attain the same configuration via a prize-sharing rule, this should be her first best choice. In short, the leader only needs to control a single type of individuals if the valuations of the prize are the same within a group. Then a single parameter, either $\delta_{i}$ or $\theta_{i}$, is sufficient to attain the first-best.

Unfortunately, this equivalence result is not robust to intra-group heterogeneity. By using the solved equilibrium with cost sharing, it is possible to confirm that the equilibrium configuration of individual contributions in a group could not be induced by prize-sharing rules. Consider for simplicity the example where $B(q, G)=q+G$ and $c_{i}(a)=\frac{a^{2}}{2}$ for all $i=1, \cdots, m$. Then, denoting $v_{i k}=\frac{M}{N_{i}}+P_{i k}$, 
the relation between the contribution of individual $k$ of group $i$ and the aggregate group effort $A_{i}$ in a cost- sharing contest is given by the equation $a_{i k}=\frac{v_{i k}}{\sum_{h=1}^{N} v_{i h}} A_{i}$. The equilibrium discount factor of marginal effort cost coincides with the HHI, as shown in equation (8).

Suppose that a prize-sharing rule of group $i$ can induce the same configuration of contributions of the individuals under the equilibrium cost-sharing rule. Condition (10) can be written as

$$
\frac{\sum_{j \neq i} A_{j}}{\left(\sum_{j=1}^{m} A_{j}\right)^{2}}\left\{\left(\theta_{i} \cdot \frac{a_{i k}}{A_{i}}+\left(1-\theta_{i}\right) \cdot \frac{1}{N_{i}}\right) \cdot M+P_{i k}\right\}+\frac{1}{\sum_{j=1}^{m} A_{j}} \cdot\left(1-\frac{a_{i k}}{A_{i}}\right) \cdot \theta_{i} M-a_{i k}=0,
$$

for such a sharing rule. Since it induces the same contributions from the individuals in the group, the values of $A_{i}$ and $\sum_{j=1}^{m} A_{j}$ are kept at the levels in the equilibrium of the cost-sharing contest. Therefore $\theta_{i}$ must satisfy the equation

$$
\begin{aligned}
\left(1-\pi_{i}\right)\left\{\left(\theta_{i} \cdot X_{i k}+\left(1-\theta_{i}\right) \cdot \frac{1}{N_{i}}\right) \cdot M+P_{i k}\right\} & +\left(1-X_{i k}\right) \cdot \theta_{i} M \\
& =\left(\sum_{j=1}^{m} A_{j}\right) \cdot a_{i k}=\frac{\left(1-\pi_{i}\right)}{\gamma_{i}^{*}} \cdot v_{i k},
\end{aligned}
$$

where $X_{i k}=\frac{v_{i k}}{\sum_{h=1}^{N} v_{i h}} \in(0,1), \quad \pi_{i}=\frac{A_{i}}{\sum_{j=1}^{m} A_{j}} \in(0,1)$, and $\gamma_{i}^{*}$ is group $i$ 's HHI on the distribution of prize valuations. ${ }^{24}$ Hence the equation

$$
\left\{1-\frac{1}{N_{i}}-\pi_{i}\left(X_{i k}-\frac{1}{N_{i}}\right)\right\} \frac{\theta_{i}}{v_{i k}}=\left(\frac{1}{\gamma_{i}^{*}}-1\right) \cdot \frac{1-\pi_{i}}{M}
$$

must hold. But then, as the individual has a larger valuation of the prize, $\theta_{i}$ has to increase to attain the effort under the equilibrium cost-sharing rule. This conclusion contradicts the construction of a prize-sharing rule (note that an increase in $v_{i k}$ increases $X_{i k}$ ).

Hence, when the valuations of the prize are different in a group, there may not exist any prize-sharing and cost-sharing rules that result in the same equilibrium configuration of contributions. The difference between the two modes of sharing, cost

24 The right-hand side of the equation is derived from the equation $\frac{\sum_{j \neq i} A_{j}}{\left(\sum_{j=1}^{m} A_{j}\right)^{2}} v_{i k}-\gamma_{i}^{*} \cdot a_{i k}=0$, which is the special case of the equilibrium condition (2). 
sharing and prize sharing, is substantial if the competing groups are characterized by intra-group heterogeneity in valuations of the prize. Both modes of sharing rules have only one control variable, which is not enough to optimally control multiple types of individuals having different valuations of the prize. The attainable result is after all the second-best, by either the cost sharing or prize sharing rules. The coincidence of these two second-best results is not generally guaranteed. It is an open question which mode of sharing is a more beneficial way for a group to provide selective incentives.

\section{Conclusion.}

Each group in a contest has to confront and manage its own collective-action problem, and cost sharing is a possible means of resolution. It is applicable not only to the case of private good prize, but also to group-specific public-good prize, a mixture of public and private good prize, and group-specific commons prize. Transfer rules in a competing group can be interpreted as the device of cost sharing in a broad class of contest situations.

We have several simple results on cost sharing in the case of intra-group homogeneity. The optimal cost-sharing rule is full-cost sharing, and it results in the same efficient configuration of contributions, as under prize sharing, by individuals belonging to the group. Once intra-group heterogeneity is introduced, such regularity is broken down. Inefficiency caused by voluntary contributions of heterogeneous members would make partial cost sharing optimal, and prize sharing would induce different contributions relative to cost sharing.

Our analysis suggests that, in a contest with cost sharing, intra-group heterogeneity is a disadvantage. It prevents the leader of a group from providing strong selective incentives to the members, and results in a low win probability. The arguments made in Nitzan and Ueda (2013) show that intra-group heterogeneity can be advantageous or disadvantageous for a group in terms of the win probability, depending on the situation it confronts (the number of contestants, toughness of the opponents and so on). Once the possibility of cost sharing is taken into consideration, however, it could add some advantage to a homogeneous group enhancing its win probability.

The degree of heterogeneity of the contested prize valuations depends on the nature of the prize and on the characteristics of the environment of the contestants. The prize valuations of group members are apparently more heterogeneous in contests 
on a public good relative to contests on a private good with equal prize sharing within the winning-group members; the distributed private-good prize could be easily changeable to money, but the benefit from a public-good prize is not. The valuations of a public-good prize would tend to be more heterogeneous in developed, secular, pluralistic open societies. Also, the valuations of a public-good prize used for more basic purposes in less developed societies would tend to be more homogeneous. Given that more unequal valuations of the contested prize within a group tend to result in a lower degree of cost sharing, we may predict, for example, that in a contest among academic institutes with reputation being the only contested public-good prize, cost sharing will be relatively low. A higher degree of cost sharing may be expected in contests among academic institutions on a private-good prize, such as government funding, assuming equal prize sharing to the faculty members of the winning institution. The empirical validity of our results can be examined by comparing the extent of actual cost sharing in contests on public-good and private-good prizes or in contests on a public-good prize with different degrees of preference heterogeneity.

Another topic that deserves further study is the generalization of the group welfare function. If group leaders embrace non-linear group welfare functions implying distributional concerns, how does it affect the equilibrium cost-sharing rules? Are such concerns advantageous or disadvantageous in enhancing the win probability of a competing group resorting to cost sharing? The study of these problems is worth pursuing.

Finally, further comparison of cost sharing and prize sharing under intragroup heterogeneity is warranted. In particular, it is interesting to identify the conditions that justify the selection by a group of cost sharing rather than prize sharing. In addition, it is important to identify the conditions justifying the simultaneous (optimal) use of both sharing modes. A model of collective contests with prize-sharing rules affording intra-group heterogeneity seems to be a prerequisite for such research. 


\section{Appendix}

\section{Proof of Proposition 1}

Define $A_{i}^{F}(t)$ for each $j=1, \cdots, m$ and $t>0$ as follows;

$$
\frac{t}{\left(t+A_{i}^{F}(t)\right)^{2}} \cdot v_{i k}=\frac{1}{N_{i}} c_{i}^{\prime}\left(a_{i k}^{F}\right) \text { for all } k=1, \cdots, N_{i} \text { and } \sum_{k=1}^{N_{i}} a_{i k}^{F}=A_{i}^{F}(t) .
$$

We can see that such a value uniquely exists. By the implicit function theorem, $A_{i}^{F}(t)$ is a continuous function of $t$. Similarly, define $A_{i}^{L}(t)$ as a continuous function of $t$ by the conditions

$$
\frac{t}{\left(t+A_{i}^{L}(t)\right)^{2}} \cdot v_{i k}=c_{i}^{\prime}\left(a_{i k}^{L}\right) \text { for all } k=1, \cdots, N_{i} \text { and } \sum_{k=1}^{N_{i}} a_{i k}^{L}=A_{i}^{L}(t) .
$$

Consider a generalized game played by the leaders with the aggregate group effort $A_{i}$ as the strategy. The strategy set of leader $i$ is $\left[A_{i}^{L}\left(\sum_{j \neq i} A_{j}\right), A_{i}^{F}\left(\sum_{j \neq i} A_{j}\right)\right]$, and the payoff function is

$$
\frac{A_{i}}{\sum_{j=1}^{m} A_{j}} \sum_{k=1}^{N_{i}} v_{i k}-E_{i}\left(A_{i}\right) .
$$

With the assumed regularity condition, we can see that this normal form satisfies standard conditions ensuring existence of an equilibrium configuration (see, for example, Friedman (1986)). Denote such a configuration by $\left(A_{1}^{*}, \cdots, A_{m}^{*}\right)$. By construction of the strategy set for this generalized game, leader $i$ can induce $A_{i}^{*}$ from the group members to satisfy (2), by choosing an adequate degree of cost sharing $\delta_{i}^{*}$. Let $\left(\delta_{1}^{*}, \cdots, \delta_{m}^{*}\right)$ be the choices by the leaders in our original model of a contest with cost sharing, and let each individual's belief be that the cost sharing rules in the other groups are those in $\left(\delta_{1}^{*}, \cdots, \delta_{m}^{*}\right)$, independently of what rule is announced by his own leader. Then each individual actually chooses a contribution that satisfies (2). Hence we have a pure-strategy perfect Bayesian equilibrium.

Q.E.D.

\section{Proof of Proposition 2}

Remember the definition of the distorted group costs, $E_{i}\left(A_{i}\right)=\sum_{k=1}^{N_{i}} c_{i}\left(a_{i k}\left(A_{i}\right)\right)$, $\sum_{k=1}^{N_{i}} a_{i k}\left(A_{i}\right)=A_{i}$ and $\frac{1}{v_{i k}} \cdot c_{i}^{\prime}\left(a_{i k}\left(A_{i}\right)\right)=\frac{1}{v_{i 1}} \cdot c_{i}^{\prime}\left(a_{i 1}\left(A_{i}\right)\right)$ for all $k=1, \cdots, N_{i}$. By using the 
latter two conditions, we have $\sum_{k=1}^{N_{i}} \frac{d a_{i k}\left(A_{i}\right)}{d A_{i}}=1$ and $\frac{d a_{i k}\left(A_{i}\right)}{d A_{i}}=\frac{v_{i k}}{v_{i 1}} \cdot \frac{c_{i}^{\prime \prime}\left(a_{i 1}\left(A_{i}\right)\right)}{c_{i}^{\prime \prime}\left(a_{i k}\left(A_{i}\right)\right)} \cdot \frac{d a_{i 1}\left(A_{i}\right)}{d A_{i}}$. We therefore obtain that

$$
\frac{d a_{i k}\left(A_{i}\right)}{d A_{i}}=\frac{\frac{v_{i k}}{c_{i}^{\prime \prime}\left(a_{i k}\left(A_{i}\right)\right)}}{\sum_{p=1}^{N_{i}} \frac{v_{i p}}{c_{i}^{\prime \prime}\left(a_{i p}\left(A_{i}\right)\right)}} \text { for all } k=1, \cdots, N_{i} .
$$

If the leader can choose $A_{i}$ without violating the constraint on $\delta_{i}$, the first-order condition

$$
0=\frac{\sum_{j \neq i} A_{j}}{\left(\sum_{j=1}^{m} A_{j}\right)^{2}} \sum_{k=1}^{N_{i}} v_{i k}-E_{i}^{\prime}\left(A_{i}\right)=\frac{\sum_{j \neq i} A_{j}}{\left(\sum_{j=1}^{m} A_{j}\right)^{2}} \sum_{k=1}^{N_{i}} v_{i k}-\sum_{k=1}^{N_{i}} c_{i}^{\prime}\left(a_{i k}\right) \cdot \frac{\frac{v_{i k}}{c_{i}^{\prime \prime}\left(a_{i k}\left(A_{i}\right)\right)}}{\sum_{p=1}^{N_{i}} \frac{v_{i p}}{c_{i}^{\prime \prime}\left(a_{i p}\left(A_{i}\right)\right)}}
$$

holds. Since the contribution of each individual belonging to the group satisfies the equation

$$
\frac{\sum_{j \neq i} A_{j}}{\left(\sum_{j=1}^{m} A_{j}\right)^{2}} \cdot v_{i k}=\gamma_{i} \cdot c_{i}^{\prime}\left(a_{i k}\left(A_{i}\right)\right) \text { for all } k=1, \cdots, N_{i},
$$

the desired equation (5) can be obtained applying some simple calculations.

Q.E.D.

\section{Proof of Proposition 3}

When $\quad c_{i}(a)=\frac{K_{i}}{1+\alpha_{i}} a^{1+\alpha_{i}}$, condition (3) implies that $a_{i k}\left(A_{i}\right)=\left(\frac{v_{i k}}{v_{i 1}}\right)^{\frac{1}{\alpha_{i}}} \cdot a_{i 1}\left(A_{i}\right)$. Since $c_{i}^{\prime \prime}\left(a_{i k}\left(A_{i}\right)\right)=K_{i} \alpha_{i} \cdot\left(\frac{v_{i k}}{v_{i 1}}\right)^{\frac{\alpha_{i}-1}{\alpha_{i}}} \cdot a_{i 1}\left(A_{i}\right)^{\alpha_{i}-1}$, we obtain (7) by substituting $c_{i}^{\prime \prime}\left(a_{i k}\left(A_{i}\right)\right)$ into (5).

Q.E.D.

\section{Proof of Proposition 5}

Since the contributions induced under a cost sharing rule is same as in the original model, we have (A1) as the relation between each individual's contribution and the aggregate effort intended by the group leader. But the summed group cost in the associated contest is defined by 


$$
E_{i}\left(A_{i}\right)=\sum_{k=1}^{N_{i}} \frac{1}{v_{i k}} \cdot c_{i}\left(a_{i k}\left(A_{i}\right)\right)
$$

The leader chooses the level of aggregate group effort to maximize

$$
\frac{A_{i}}{\sum_{j=1}^{m} A_{j}} N_{i}-E_{i}\left(A_{i}\right) \text {. }
$$

The first-order condition is

$$
0=\frac{\sum_{j \neq i} A_{j}}{\left(\sum_{j=1}^{m} A_{j}\right)^{2}} N_{i}-E_{i}^{\prime}\left(A_{i}\right)=\frac{\sum_{j \neq i} A_{j}}{\left(\sum_{j=1}^{m} A_{j}\right)^{2}} N_{i}-\sum_{k=1}^{N_{i}} \frac{c_{i}^{\prime}\left(a_{i k}\right)}{v_{i k}} \cdot \frac{\frac{v_{i k}}{c_{i}^{\prime \prime}\left(a_{i k}\left(A_{i}\right)\right)}}{\sum_{p=1}^{N_{i}} \frac{v_{i p}}{c_{i}^{\prime \prime}\left(a_{i p}\left(A_{i}\right)\right)}} .
$$

Since the contribution of each individual belonging to the group satisfies the equation

$$
\frac{\sum_{j \neq i} A_{j}}{\left(\sum_{j=1}^{m} A_{j}\right)^{2}}=\gamma_{i} \cdot \frac{1}{v_{i k}} \cdot c_{i}^{\prime}\left(a_{i k}\left(A_{i}\right)\right) \text { for all } k=1, \cdots, N_{i},
$$

we have $\gamma_{i}=\frac{1}{N_{i}}$.

Q.E.D. 


\section{References}

Baik, K. H. 1994. "Winner-Help-Loser Group Formation in Rent-Seeking Contests." Economics and Politics, 6(2): 147-62.

Baik, Kyung H. 2008. “Contests with Group-Specific Public-Good Prizes.” Social Choice and Welfare, 30(1): 103-117.

Baik, Kyung H., and Sanghack Lee. 2007. “Collective Rent Seeking When Sharing Rules are Private Information.” European Journal of Political Economy 23 (September): 768776.

Cornes, Richard and Roger Hartley. 2005. "Asymmetric Contests with General Technologies.” Economic Theory 26(4), 923-946.

Dasgupta, Partha, Amartya Sen, and David Starrett. 1973. "Notes on the Measurement of Inequality.” Journal of Economic Theory, 6(2): 180-187.

Esteban, Joan-Maria, and Debraj Ray. 2001. "Collective Action and the Group Size Paradox.” American Political Science Review 95 (September): 663-72.

Esteban, Joan-Maria, and Debraj Ray. 2011. “A Model of Ethnic Conflict.” Journal of the European Economic Association. 9(3): 1496-1521.

Friedman, James W. 1986. Game Theory with Applications to Economics. New York: Oxford University Press.

Fudenberg, Drew and Jean Tirole. 1991. "Perfect Bayesian Equilibrium and Sequential Equilibrium.” Journal of Economic Theory 53 (2): 236-260.

Hillman, Arye L. and John G. Riley. 1989. "Politically Contestable Rents and Transfers.” Economics and Politics 1 (March): 17-39.

Katz, Eliakim, Shmuel Nitzan, and Jacob Rosenberg. 1990. "Rent-Seeking for Pure Public Goods.” Public Choice 65 (April): 49-60.

Katz, Michael L. 1991. “Game-Playing Agents: Unobservable Contracts as Precommitments.” Rand Journal of Economics 22 (Autumun): 307-28

Kolmar, Martin and Hendrik Rommeswinkel. 2013. "Contests with Group-Specific Public Goods and Complementarities in Efforts.” Journal of Economic Behavior and Organization 89: 9-22

Konrad, Kai A. 2009. Strategy and Dynamics in Contests. New York: Oxford University Press.

Lee, Sanghack. 1995. "Endogenous Sharing Rules in Collective-Group Rent-Seeking.” Public Choice 85 (October): 31-44. 
Lehmer, Derrick H. 1971. “On the Compounding of Certain Means.” Journal of Mathematical Analysis and Applications 36: 183-200.

Nitzan, Shmuel. 1991. "Collective Rent Dissipation. Economic Journal 101 (November):1522-1534.

Nitzan, Shmuel, and Kaoru Ueda. 2009. "Collective Contests for Commons and Club Goods.” Journal of Public Economics, 93 (1), 48-55.

Nitzan, Shmuel, and Kaoru Ueda. 2011. "Prize Sharing in Collective Contests.” European Economic Review, 55(5), 678-687.

Nitzan, Shmuel, and Kaoru Ueda. 2013. "Intra-group Heterogeneity in Collective Contests.” Social Choice and Welfare, downloadable at SpringerLink.

Olson, Mancur. 1965. The logic of collective action. Cambridge, M.A., Harvard University Press.

Riaz, Khalid, Jason F. Shogren and Stanley R. Johnson. 1995. “A General Model of RentSeeking for Public Goods.” Public Choice 82 (March): 243-59.

Ryvkin, Dmitry. 2011. "The optimal sorting of players in contests between groups." Games and Economic Behavior, 73(2): 564-572.

Sen, Amartya K. 1966. “Labour Allocation in a Cooperative Enterprise.” Review of Economic Studies 33 (October): 361-71.

Ueda, Kaoru. 2002. “Oligopolization in Collective Rent-Seeking.” Social Choice and Welfare 19 (July): 613-26.

Vázquez, Álex. 2014. "Sharing the effort costs in collective contests.” Available at SSRN: http://ssrn.com/abstract=2378042 or http://dx.doi.org/10.2139/ssrn.2378042. 